\title{
OTIMIZAÇÃO E REDIMENSIONAMENTO DA SEDIMENTAÇÃO DO REJEITO DE MINÉRIO FERRO
}

\section{OPTIMIZATION AND SIZING OF IRON ORE WASTE SEDIMENTATION}

\author{
T. M. F.S. de OLIVEIRA ${ }^{1}$, F. A . B. CALDEIRA ${ }^{1}$, E. C. CALDEIRA ${ }^{1}$, L.D. RIBEIRO ${ }^{1}$ e A. B. REIS ${ }^{*}$ \\ ${ }^{1}$ UFJM - Universidade Federal dos Vales do Jequitinhonha e Mucuri, Engenharia Química, Diamantina-MG/Brasil
}

\author{
${ }^{*}$ Corresponding author. Universidade Federal dos Vales do Jequitinhonha e Mucuri, Instituto de Ciência e Tecnologia, Diamantina, Minas Gerais, \\ Brasil, +5538991279624 \\ e-mail addressl:arlete.reis@ict.ufvjm.edu.br(A.B.Reis)
}

\begin{tabular}{l} 
A R T I C L E I N F O \\
\hline Article history: \\
Received 2020-06-30 \\
Accepted 2020-10-20 \\
Available online 2020-10-20 \\
pa l a v r a s - ch a ve \\
Mineração \\
Reaproveitamento \\
Lei de stokes \\
ke yw ords \\
Mining \\
Reuse \\
Stokes Law
\end{tabular}

\begin{abstract}
A B S T R A C T
Sedimentation is widely used in industry, from recycling to reuse in the mining industry, where iron ore tailings are present in the sedimentation sludge. It aimed to analyze the $\mathrm{pH}$ and concentration of ore tailings in the sedimentation, to compare the characteristics of the particles in the sedimentation. He applied the complete $2^{2}$ batch factorial design, then analyzed the concentration of tailings particles in the sludge and in the clarified one. Calculations of the parameters related to sedimentation were performed. It designed a didactic sedimentation module, with a capacity of $1 L$. The best sedimentation was in acidic to neutral pH, by Scanning Electron Microscopy observed the influence of the particle diameter, providing even the possibility of optimization in water recovery processes, in the sedimentation of the mining industry.
\end{abstract}

\footnotetext{
R E S U M O

A sedimentação é muito empregada na indústria, desde a reciclagem até o reuso na indústria de mineração, onde há presença de rejeito de minério de ferro na lama de sedimentação. Objetivou analisar o pH e concentração do rejeito de minério na sedimentação, comparar as características das partículas na sedimentação. Aplicou o planejamento fatorial completo $2^{2}$ em batelada, em seguida, analisou a concentração de partículas de rejeito no lodo e no clarificado. Foram realizados cálculos dos parâmetros relacionado a sedimentação. Dimensionou um módulo didático de sedimentação, com capacidade de um $1 \mathrm{~L}$. A melhor sedimentação foi em $\mathrm{pH}$ de ácido para neutro, pela Microscopia Eletrônica de Varredura observou a influência do diâmetro de partícula, propiciando inclusive a possibilidade de otimização em processos de recuperação de água, na sedimentação da indústria de mineração.
} 


\section{INTRODUÇÃO}

A mineração é considerada como uma das atividades industriais com maior potencial poluidor. Paralelamente, a legislação ambiental em muitos países é frágil, o que gera diversos conflitos entre a sociedade e a iniciativa privada. Isso provoca, na maioria das vezes, degradação ambiental, resultando em grandes problemas ambientais associados à atividade mineradora (FONSECA, 2008). No intuito de minimizar os resíduos da mineração, paralelo à possibilidade de criação e desenvolvimento de novos produtos, a reciclagem apresenta-se como uma das formas atender tanto as questões de proteção ambiental quanto as empresas pois pode gerar valor econômico solucionando problemas de gerenciamento de resíduos (CABRAL e MORIS, 2010).

Para a viabilização da reciclagem, é necessário utilizarse de operações unitárias, como por exemplo, a sedimentação (decantação), a qual é muito empregada em processos químicos industriais e bastante utilizada na remoção de partículas sólidas em suspensão (NUNES, 2008). A sedimentação pode ainda, de acordo com o interesse industrial, ter grande potencial de aplicação, no reaproveitamento de produto de qualquer uma de suas distintas fases: fase lodo e fase clarificado, correspondente às fases sólida e líquida, respectivamente.

$\mathrm{Na}$ sedimentação, as fases sólidas e líquidas são separadas devido à ação da gravidade e pela diferença de densidade da partícula e da solução a qual está inserida (NUNES, 2008). Dessa forma, tanto na fase sólida, quanto na fase líquida, pode-se obter produto com viabilidade de reaproveitamento, sendo estes, o sólido concentrado e o líquido com aspecto límpido, ambos obtidos a partir das respectivas fases de espessamento e clarificação.

Dessa forma, objetivou-se sedimentar amostras de rejeito de minério de ferro, cedidos pela mineradora Samarco Mineração S.A, primeiramente em batelada com provetas de $250 \mathrm{~mL}$ e posteriormente, redimensionando para o módulo de didático de sedimentação com volume de 1000 mL, mantendose concentração e pH adequado. Em seguida, foram calculados os parâmetros: concentração da amostra nas fases clarificado e lodo, densidade da partícula, número de Reynolds, coeficiente de arraste, diâmetro de Stokes, velocidade de partícula e velocidade de sedimentação.

\section{METODOLOGIA}

A amostra de rejeito de minério de ferro foi cedida pela empresa Samarco Mineração S.A, na forma sólida (pó). Os experimentos em batelada, foram realizados em triplicata, de modo a viabilizar, com maior eficiência, as variáveis que interferem na sedimentação, essa etapa de otimização foi de crucial importância para o redimensionamento em módulo didático.

O rejeito passou apenas por um processo de peneiramento, utilizando-se a peneira de abertura de 10 mesh, simulando situação verdadeira. Para o processo de otimização, foi utilizado um planejamento fatorial completo $2^{2}$, incluindo os 4 pontos axiais e 4 pontos centrais para avaliação do erro puro, método descrito por Rodrigues e Lemma (2009), totalizando 12 ensaios. As variáveis empregadas no sistema foram o $\mathrm{pH}$ e a concentração de rejeito, sem adição de agente floculante, variando pH 4,9 a 9,12 e concentração de rejeito de 28,9 a 71,2 $\%(\mathrm{~m} / \mathrm{m})$.

As doze amostras foram sedimentadas por trinta minutos em seus respectivos pH e concentração de rejeito, em seguida, foi realizada a leitura da turbidez. Com os melhores resultados, plotou-se o gráfico da altura do sedimentado $(\mathrm{cm})$ versus tempo (minutos), em seguida, foi calculada a concentração de rejeito no clarificado e no lodo. Para determinar a concentração de sólidos no clarificado e no lodo, pesou-se a placa de Petri com amostra e placa com o sólido tanto para o clarificado, quanto para o lodo, na sequência secas em estufa de secagem na temperatura de $104{ }^{\circ} \mathrm{C}$, por um período de $24 \mathrm{hrs}$, e pesados em seguida. Com esses dados, foi possível determinar a concentração de sólidos suspensos $\left(\mathrm{C}_{\mathrm{ss}}\right)$.

Para melhor entender a sedimentação envolvendo a relação entre o $\mathrm{pH}$ e a concentração de amostras foram feitas fotos por $\mathrm{MEV}$, no qual buscou analisar as características das partículas envolvidas no processo, por isso analisou as amostras do clarificado e do lodo dos pH's ácido, neutro e básico, sendo eles 5,5, 7,0 e 9,12 respectivamente. A partir dessas fotos foi possível aferir o diâmetro médio das partículas da melhor sedimentação.

Em seguida, foi determinada a densidade da amostra pelo método de picnometria, onde, foram realizadas pesagens (g) do picnômetro vazio, do picnômetro com água e do picnômetro com água mais amostra de rejeito de minério de ferro.

A partir dos dados da densidade e do diâmetro da partícula, foram realizados os cálculos dos parâmetros: de Reynolds, coeficiente de arraste, diâmetro de Stokes, velocidade de partícula e velocidade de sedimentação, conforme ilustram as equações de 1 a 4 respectivamente (MASSARANI, 2002), para fim de entender melhor a sedimentação da partícula e velocidade de sedimentação. Assim, foi redimensionado para o módulo didático de sedimentação, com capacidade de armazenagem de 1 litro, onde foi realizada a reprodutibilidade dos experimentos de sedimentação.

$$
\begin{aligned}
& R_{e}=\frac{\rho_{p} V_{s} d_{p}}{\mu} \\
& C_{D}=\frac{24}{R_{e}} \\
& V_{p}=\frac{2 \cdot r^{2} \cdot g \cdot\left(\rho_{p}-\rho_{f}\right)}{9 \mu} \\
& V_{s}=\sqrt{\frac{4 \cdot\left(\rho_{p}-\rho_{f}\right) \cdot g \cdot(2 r)}{3 \cdot C_{D} \cdot \rho_{f}}}
\end{aligned}
$$

onde: $R e$ - número de Reynolds; $\mathrm{V}_{\mathrm{s}}$ - velocidade de sedimentação; $\rho_{p}$ - densidade da partícula; $d_{p}-$ diâmetro da partícula; $\mu$ - viscosidade do fluido; $\mathrm{V}_{\mathrm{s}}$ - velocidade de sedimentação; $r$ - raio da partícula; $g$ - gravidade; $\rho_{\mathrm{f}}$ - densidade do fluido. 


\section{RESULTADOS E DISCUSSÃO}

Analisar as variáveis de um processo é de grande importância para otimização, redução de custos e melhoria na produção final, por isso, analisou-se as concentrações de rejeito de minério e $\mathrm{pH}$ na sedimentação em proveta de 250 $\mathrm{mL}$, utilizando o planejamento fatorial. De acordo com a Figura 4 , podemos inferir que a região de $\mathrm{pH}$ ótimo varia entre 5,0 até 7,0 e a concentração variou de 20 a $70 \%$, sendo que a melhor sedimentação foi em pH 6,85 e concentração $50 \%$, apresentando no clarificado, uma turbidez de 111 NTU, em 30 minutos de sedimentação. Observando a Figura 2, é notório a verificação do clarificado mais claro em pH's mais baixo, ao contrário dos pH's básicos.

Na Figura 1 apresentou um $\mathrm{R}^{2}$ de $96,98 \%$, sendo um ótimo resultado, verificando que os dados obtidos apresentam um grande índice de confiança. A variação na faixa de $\mathrm{pH}$ e de concentração é um ponto positivo, uma vez que em processo continuo essas variáveis podem sofrer mudança, demonstrando uma região de segurança.
Figura1 - Planejamento fatorial variando pH e concentração do rejeito de minério.

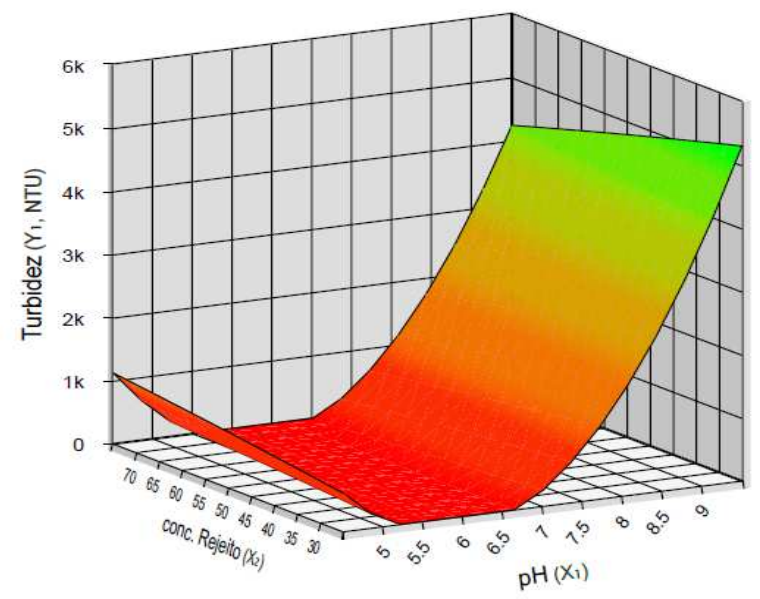

Figura 2 - Sedimentação em proveta diferentes pH e concentração de rejeito

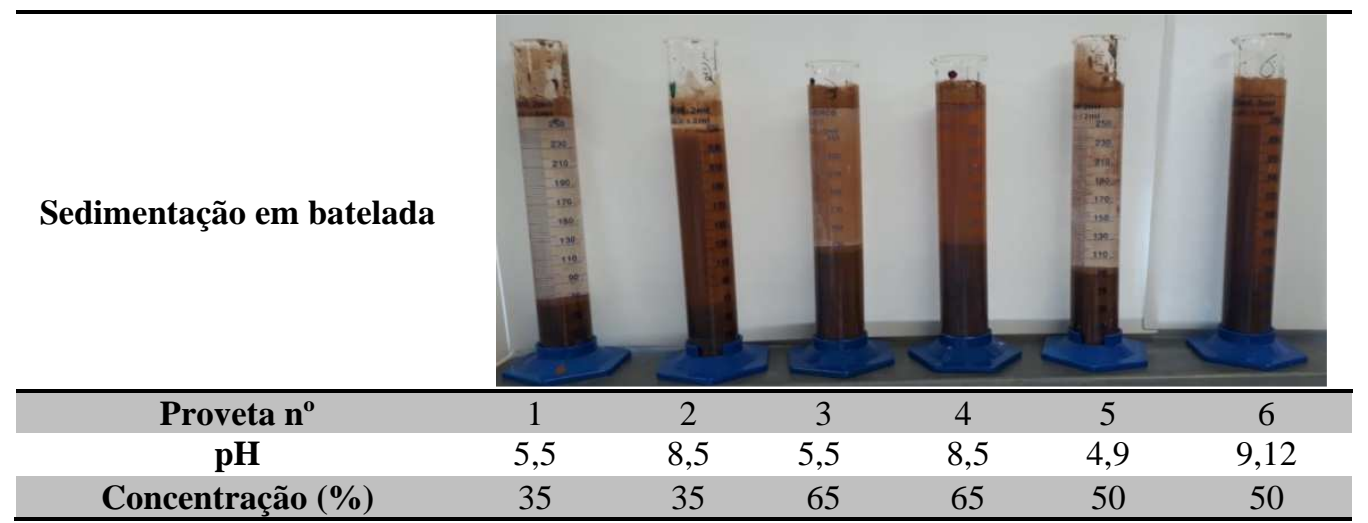

Em seguida, foram verificadas as concentrações de rejeito no clarificado e no lodo, no clarificado apresentou $0,00037 \mathrm{~g} / \mathrm{cm}^{3}$ sólido seco já no lodo apresentou $0,571 \mathrm{~g} / \mathrm{cm}^{3}$ sólido seco, esses dados demonstram que o clarificado apresenta baixa concentração em relação ao lodo, mais de 1500 vezes, indicando que o método de sedimentação apresentou grande eficiência, conforme ilustra a Figura 2.

Analisando as imagens de MEV (Figuras 3 1L,1C, 2L, 2C, 3L, 3C) o pH que apresentou o melhor clarificado foi do ácido para neutro, representados na Figura 3 como 1 e 2 respectivamente. Pode-se observar que as particulas presentes no lodo apresentaram maiores diâmetros que as partículas presentes no clarificado e, em ambiente básico $(\mathrm{pH} \mathrm{9,12)} \mathrm{as} \mathrm{partículas} \mathrm{do} \mathrm{lodo} \mathrm{apresentaram} \mathrm{diâmetro}$ menor nos ambiente ácidos $(\mathrm{pH} 5,5)$ e neutros $(\mathrm{pH} 7,0)$, confirmando que o diâmetro da particula tem influência direta na sedimentação, visto que quanto maior a partícula, melhor será a sedimentação devido a ação gravitacional, e nesse caso, os melhores resultados foram em pH's de ácidos a neutros.

Com as imagens do MEV obteve-se o diâmetro das particulas do lodo de $57,06 \mu \mathrm{m}$ e densidade de $1,0051 \mathrm{~g} / \mathrm{cm}^{3}$, foi possível determinar o número de Reynolds de $6,68 \times 10^{-5}$. Como $\operatorname{Re}<1$, o regime se adequa ao de Stokes, segundo SILVA (2006), e, a partir de então foi possível obter um coeficiente de arraste de 359053,44. O diâmetro de Stokes apresentou $1,60 \times 10^{-5} \mathrm{~m}$, a velocidade da partícula de $9,11 \times 10^{-8} \mathrm{~m} / \mathrm{s}$ e velocidade de sedimentação de $0,0001 \mathrm{~m} / \mathrm{s}$. 
Figura 3 - Imagens de MEV para a fase Lodo (1L (pH 5,5), 2L (pH 7,0) e 3L (pH 9,12) e para a fase clarificado (1C (pH $5,5), 2 \mathrm{C}(\mathrm{pH} 7,0)$ e 3C (pH 9,12)), ampliado 500 vezes.
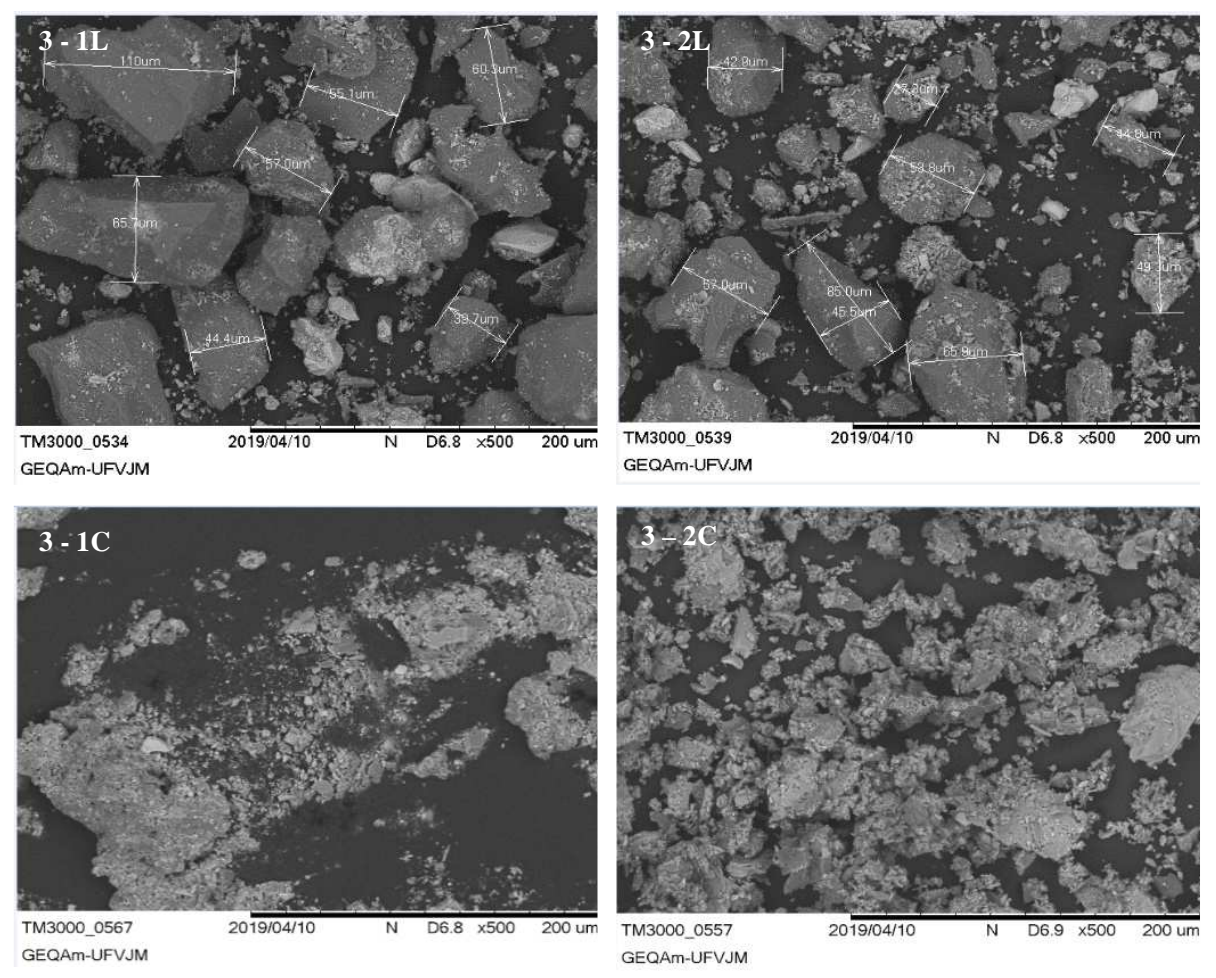
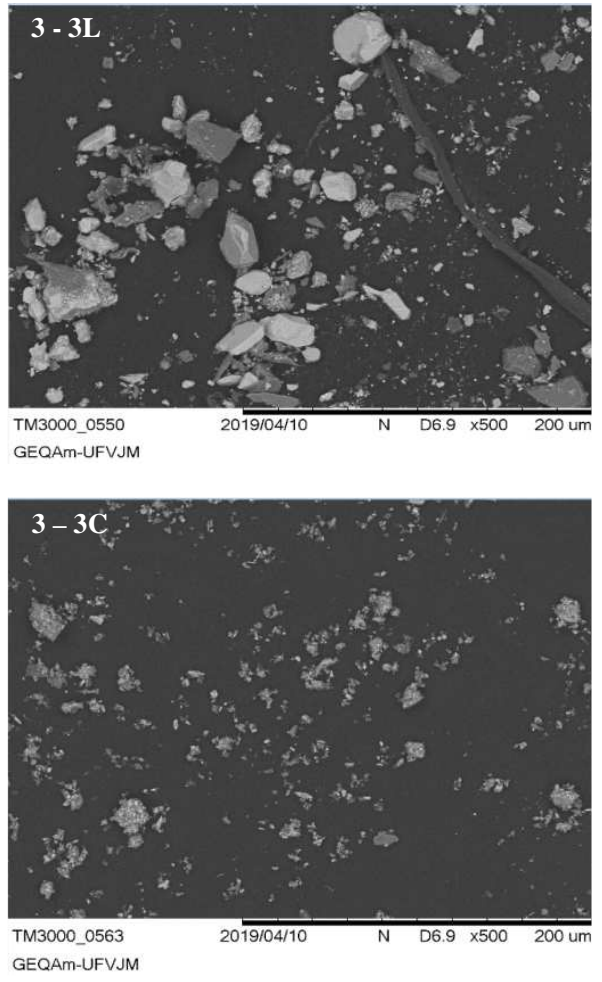

Assim, foi possível redimensionar a operação de sedimentação para um módulo didático, com capacidade para $1000 \mathrm{~mL}$, no $\mathrm{pH} 6,85$ e concentração de $50 \%$ de rejeito de minério, que de acordo com o Figura 4, a sedimentação com a proveta durou 36 minutos, já no módulo foi com 70 minutos, quase o dobro de tempo gasto na proveta, no entanto, o volume aumentado de um para o outro foi de 4 vezes, então o tempo de sedimentação pode ser considerável como aceitável, além disso, os dois gráficos apresentaram $\mathrm{R}^{2}$ de 0,999, sendo um índice de confiança muito bom. A turbidez do clarificado foi de 133 NTU, o que implica em afirmar que houve um aumento de 22 NTU. Analisando a velocidade de sedimentação, com proveta obteve velocidade $0,0001 \mathrm{~m} / \mathrm{s}$, com o módulo $0,00007 \mathrm{~m} / \mathrm{s}$, comprovando a velocidade maior de sedimentação na proveta.

Figura 4 - Sedimentação do rejeito em proveta e em módulo didático
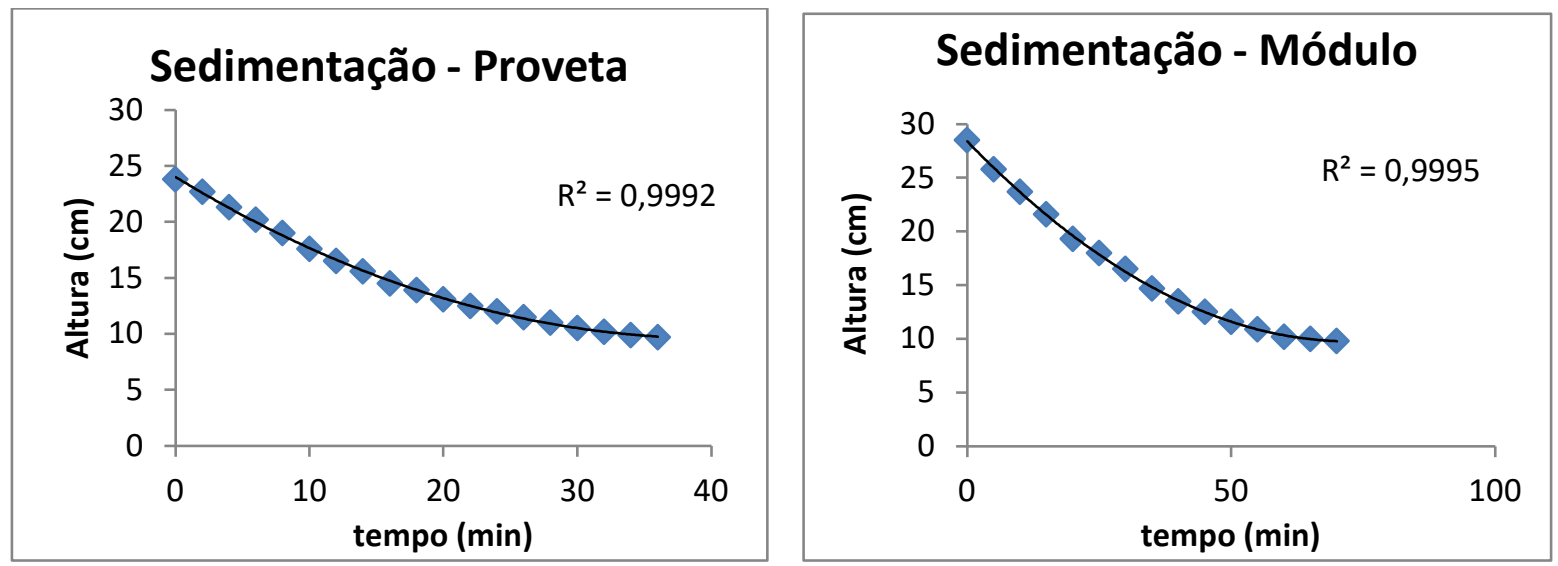


\section{CONCLUSÃO}

Nos ensaios com as provetas pode-se concluir que a melhor sedimentação acontece em ambiente com $\mathrm{pH}$ de ácido a neutro, com concentrações baixas a elevadas de rejeito, dado considerado de elevada importância, considerando que mudanças bruscas de concentração podem acontecer sem que haja perca de eficiência. No MEV obtevese o diâmetro médio da partícula de $57,06 \mu \mathrm{m}$, e observou que as partículas com melhor sedimentação apresentam maiores diâmetros, sofrendo mais com o efeito da gravidade e, assim, sedimentando. A concentração de rejeito no clarificado apresentou um percentual muito menor que no lodo, mais de 1500 vezes. Além disso, com o número de Reynolds de $6,68 \times 10^{-5}$, foi possível concluir que o escoamento se encontra num intervalo que o classifica no regime de Stokes, direcionando assim, a sequência de cálculos que nos permitiu determinar o coeficiente de arraste de 359053,44 . O diâmetro de Stokes foi de $1,60 \times 10^{-5} \mathrm{~m}$, a velocidade da partícula de $9,11 \times 10^{-8} \mathrm{~m} / \mathrm{s}$ e velocidade de sedimentação de $0,0001 \mathrm{~m} / \mathrm{s}$. Dessa forma, no rejeito da mineração todo material pode ser aproveitado após a sedimentação utilizando outras operações, tanto o clarificado, recuperando a água, quanto o lodo, utilizando a lama para aplicações diversas, tais como: materiais cerâmicos, reprocessamento na obtenção de minerais, aproveitamento na construção civil entre outros, assim substituindo ou desafogando a destinação final, as barragens de minério.

\section{AGRADECIMENTOS}

Os autores agradecem à FAPEMIG, projeto APQ03624-17 Rede Candongas: Aplicação de Tecnologias Desenvolvidas e Estudos de Viabilidade Técnica e Econômica para a Transformação e Valoração dos Rejeitos Dragados da Barragem de Candongas (Transformando o Desastre do Rio Doce em Oportunidades Locais). Aos laboratórios LIPEMVALE-UFVJM; Ao LMMA financiado pela FAPEMIG (CEX-112-10), SECTES/MG e RQ-MG. Ao GEPEAQ-Grupo de Estudos e Pesquisas Aplicadas à Engenharia Química.

\section{R E F E R E N C IAS}

CABRAL, M. S.; MORIS, V. A. S. Reaproveitamento da Borra de Café como Medida de Minimização da Geração de Resíduos. São Carlos, SP, Brasil, 2010.

FONSECA, D. C. A. Alternativas de reuso de água em indústrias de beneficiamento de minério. Monografia (Especialização em Engenharia e Meio Ambiente) Universidade Federal de Minas Gerais, Belo Horizonte, MG, 2008.

MASSARANI, G. Fluidodinâmica em Sistemas Particulados. $2^{\mathrm{a}}$ edição, Rio de Janeiro, E-papers Serviços Editoriais, 2002.
NUNES, J. F. Estudo Da Sedimentação Gravitacional De Suspensões Floculentas. Dissertação (Mestrado em Enhgenharia Química) - Universidade Federal de Uberlândia, Uberlândia, MG, 2008.

RODRIGUES, M. I.; LEMMA, A. F. Planejamento de Experimentos e Otimização de processos. Campinas, São Paulo: Editora Cárita, 2009.

SILVA, R. G. Estudo numérico de movimentação de partículas em escoamento. Dissertação (Mestrado em Engenharia) - Escola Politécnica da Universidade de São Paulo, São Paulo, SP, 2006. 